\title{
Pre-vascularization in fibrin Gel/PLGA microsphere scaffolds designed for bone regeneration
}

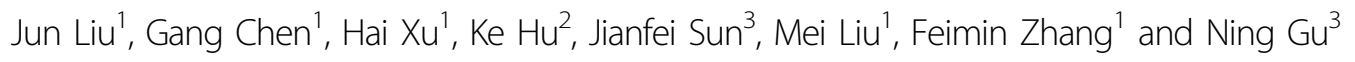

\begin{abstract}
Blood supply plays a central role in alveolar bone regeneration within a large bone defect filled with a cell-laden scaffold material, as it provides sufficient oxygen and nutrition to cells inside the scaffold. To address the issue of insufficient vascularization within scaffolds designed to promote bone regeneration, we developed a pre-vascularized scaffold to enable the repair of large alveolar bone defects. Peripheral blood-derived mesenchymal stem cells (PBMSCs) and endothelial colony-forming cells (ECFCs) were collected from peripheral blood and incorporated into fibrin gel, which was then mixed with poly(lactic-co-glycolic acid) (PLGA) microspheres to form a fibrin gel/PLGA microsphere (FP) scaffold. The induction of osteogenic differentiation of PBMSCs and the pre-vascularization in the FP scaffold were achieved separately under different conditions. PBMSCs seeded into the FP scaffolds with fibrin gel tended to migrate to the surface of PLGA microspheres and express high levels of osteogenic markers. ECFCs cocultured with PBMSCs in FP scaffolds were inclined to form a capillary-like structure in the gel substrate. These capillary-like structures penetrated the space among the microspheres and are supposed to anastomosis with blood capillaries in vivo. Together these results indicate that the pre-vascularized FP scaffold may overcome the shortages of oxygen and nutrition inside conventional scaffolds, leading to a better clinical effect.
\end{abstract}

\section{Introduction}

Alveolar bone defects have become common clinically, and the use of tissue engineering scaffolds to repair such defects has become popular in clinical practice, based on studies showing that this approach is preferable to conventional bone grafts ${ }^{1,2}$. In bone tissue engineering, scaffolds play an important role in providing threedimensional structures for cell adhesion, proliferation, and differentiation ${ }^{3-5}$, and bone regeneration depends on the proliferation and differentiation of seed cells migrating into the defect area from the surrounding tissue ${ }^{2}$. To accelerate bone regeneration, seed cells have been pre-

\footnotetext{
Correspondence: Feimin Zhang (fmzhang@njmu.edu.cn)

${ }^{1}$ Jiangsu Key Laboratory of Oral Diseases, Nanjing Medical University, 210029 Nanjing, China

${ }^{2}$ Key Laboratory of Clinical and Medical Engineering, School of Biomedical Engineering, Department of Basic Medical Sciences, Nanjing Medical

University, 210029 Nanjing, China

Full list of author information is available at the end of the article.

These authors contributed equally: Jun Liu, Gang Chen
}

seeded within scaffolds and cultured in vitro before implantation $^{6}$. For regeneration of alveolar bone though, these seed cells require adequate blood supply to provide the nutrient exchange necessary for their high metabolic rate $^{7}$. Without vascularization within the scaffold, commonly only the cells in the superficial zone of an implanted scaffold can survive, as these are the only cells that receive oxygen and nutrients diffusing from the tissue around the defect. Therefore, establishment of blood supply provide by vessels throughout scaffolds is crucial for the survival of cells deep within the scaffold ${ }^{8}$. Blood vessels can form within regenerating bone over a period of time ${ }^{9}$, and the growth rate of new blood vessels is estimated to be about $200 \mu \mathrm{m} / \mathrm{day}^{10,11}$. Thus, for a large bone defect, the long period for vascularization to occur will likely cause the cells in the interior of the scaffold to experience oxygen and nutrient deficiency, which may increase the risk of graft failure ${ }^{12-14}$.

\section{(c) The Author(s) 2018}

(c) (i) Open Access This article is licensed under a Creative Commons Attribution 4.0 International License, which permits use, sharing, adaptation, distribution and reproduction cc) in any medium or format, as long as you give appropriate credit to the original author(s) and the source, provide a link to the Creative Commons license, and indicate if changes were made. The images or other third party material in this article are included in the article's Creative Commons license, unless indicated otherwise in a credit line to the material. If material is not included in the article's Creative Commons license and your intended use is not permitted by statutory regulation or exceeds the permitted use, you will need to obtain permission directly from the copyright holder. To view a copy of this license, visit http://creativecommons.org/licenses/by/4.0/. 

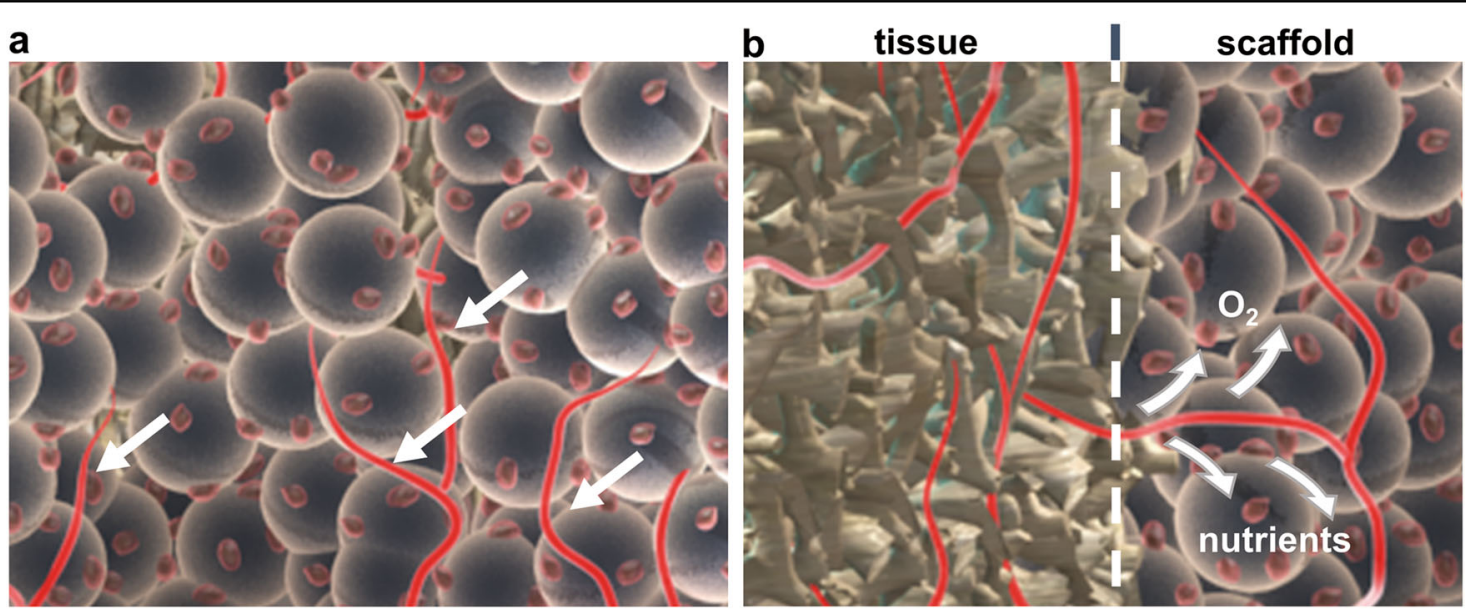

Fig. 1 Concept of pre-vascularization in FP scaffold. a Capillary-like structures in the scaffold (white arrows) and cells on the surface of PLGA microspheres. $\mathbf{b}$ Pre-vascularized scaffold after implantation in vivo. The capillary-like structures anastomose with the capillaries from the surrounding tissue and then supply oxygen and nutrients to the cells within the scaffold

Vascularization of engineered scaffolds prior to implantation may provide a promising way to solve the problem. The vessel structures already established within prevascularized scaffolds in vitro may rapidly anastomose with the host vasculature upon implantation to provide blood supply throughout the scaffold ${ }^{15,16}$. Moreover, Rao and Stegemann proposed that the presence of blood vessels may have additional benefits for bone regeneration, such as allowing the transportation of osteoprogenitor cells to the defect site to form bone tissue ${ }^{17}$. Therefore, pre-vascularization of scaffolds is a promising approach for large-scale alveolar bone regeneration. Accordingly, we designed a pre-vascularized cell/scaffold composite, in which pre-vascularized structures are distributed throughout the scaffold (Fig. 1). To achieve pre-vascularization, the selection of seed cells is important. The blood supply of alveolar bone is essentially provided by abundant capillaries. Previous studies have confirmed that endothelial cells co-cultured with mesenchymal cells in threedimensional matrixes tend to form capillary-like structures $^{18}$. Thus, we chose these cells in our strategy to induce the formation of capillary-like structures within scaffolds in vitro prior to implantation. Two types of essential cells can both be isolated from peripheral blood: endothelial colony-forming cells (ECFCs), a type of endothelial cell that can be used to form capillary-like structures in scaffolds for pre-vascularization ${ }^{19}$, and peripheral blood-derived mesenchymal stem cells (PBMSCs), which have the capabilities of rapid proliferation and osteogenic differentia$\operatorname{tion}^{20}$. Furthermore, studies have shown that the coculture of endothelial cells and mesenchymal stem cells (MSCs) can facilitate the formation of capillary-like structures and the angiogenic process ${ }^{14,18}$. Therefore, PBMSCs were selected in our study based on their potential to participate in both the processes of pre-vascularization and osteogenic differentiation.

Bioactive properties of biopolymers (collagen, hydrogels, chitosan, and fibrin, etc.), such as high hydrophilicity and biocompatibility, are conducive to cell proliferation and migration ${ }^{18,21,22}$. Moreover, most of these biopolymers can be induced to form gels under certain conditions. Promisingly, research has demonstrated that endothelial cells can form capillary-like structures spontaneously within such three-dimensional gels ${ }^{23,24}$. However, although beneficial for vascularization, a soft gel is not the best choice for osteogenic differentiation of $\mathrm{MSCs}^{25}$. As anchoragedependent cells, osteoblasts must adhere to a rigid substrate in order to retain their phenotype ${ }^{26}$. For this reason, artificial synthetic polymers, such as polyglycolic acid, polylactic acid, poly(lactic-co-glycolic acid) (PLGA), and poly( $\varepsilon$-caprolactone), have been widely studied and developed to offer the ideal properties, including a rigid surface, for osteoblast differentiation ${ }^{26-28}$.

To acquire a pre-vascularized composite for bone regeneration, fibrin gel and PLGA were adopted in the present study, as they both have been used in the clinic and share the advantages of safety, low cost, and easy availability $^{29-32}$. The two materials were processed by specific methods and integrated to from a fibrin gel/PLGA microsphere (FP) scaffold. Fibrin easily self-assembles to form a gel $^{22}$, and the fibrin gel possesses a structure similar to the extracellular matrix in vivo. The PLGA microspheres were prepared under a double emulsion-evaporation procedure and then stacked into a scaffold with high porosity and interconnected pores distributed within the scaffold. The fibrin precursor solution (fibrinogen) containing cells was mixed with the PLGA microsphere scaffold and coagulated in situ. The 
two components of the FP scaffolds had different effects on osteogenic induction and pre-vascularization. PBMSCs tended to migrate to the surface of the PLGA microspheres, whereas in the fibrin gel, capillary-like structures were formed by ECFCs. Accordingly, the FP scaffold contains different microenvironments suitable for prevascularization and bone regeneration, making it a promising tissue engineered scaffold for the repair of large alveolar bone defects.

\section{Materials and methods}

Collection and identification of cells from peripheral blood

The two cell types used in this study were collected from the peripheral blood of adult healthy New Zealand white rabbits, weighing $4.0-4.5 \mathrm{~kg}$ (Nanjing Anlimo Technology Co., Ltd, Jiangsu, China). All animal experimental procedures followed the ethical guidelines for the use of experimental animals approved by the Institutional Animal Care and Use Committee of Nanjing Medical University. First, $10-20 \mathrm{~mL}$ fresh blood was collected from the rabbit via a syringe into a tube containing $1-2 \mathrm{~mL}$ heparin sodium solution $\left(250 \mathrm{U} \mathrm{mL}^{-1}\right)$. Then, the anticoagulant/blood mixture was centrifugated with lymphocyte separation medium (TBD, Tianjin, China) for isolation of the mononuclear cells in the buffy coat. After two washes with phosphate-buffered saline (PBS) for $10 \mathrm{~min}$ each, the cells were resuspended in endothelial growth medium-2 (EGM-2, Lonza, Basel, Switzerland). The cells were pipetted into a six-well culture plate that had been coated with fibronectin (Solarbio, Beijing, China) in advance. The plated cells were incubated at $37^{\circ} \mathrm{C}$ in a humidified environment with $5 \% \mathrm{CO}_{2}$. After $24 \mathrm{~h}$, the plate was washed to remove unattached cells. The culture medium was changed daily for the next 7 days and then every 2-3 days thereafter.

From the 7th day in primary culture, colonies of ECFCs and PBMSCs with differing cellular morphologies as described by previous studies were observed under an inverted microscope (DMILLED, Leica, Germany) every other day ${ }^{19,20,33}$. The cells could be separated by the cloning cylinder method when the number of cells in a colony reached 100-200. After separation from the primary culture, ECFCs were cultured in EGM-2, and PBMSCs were cultured in complete modified $\alpha$-minimal essential medium (modified $\alpha$-MEM, HyClone, UT, USA) supplemented with $10 \%$ fetal bovine serum (FBS, HyClone) and $1 \%$ penicillin-streptomycin (HyClone). Then, they were expanded, cultured, and cryopreserved following standard cell culture procedures.

\section{Indirect immunofluorescence for ECFC and PBMSC identification}

Cells (passage 4) were fixed immediately with $4 \%$ paraformaldehyde for $30 \mathrm{~min}$ at $4{ }^{\circ} \mathrm{C}$. Permeabilization was needed for the detection of von Willebrand factor (vWF). Cells were incubated for $15 \mathrm{~min}$ in $0.25 \%$ Triton X-100. Then blocking was achieved by incubation in $10 \%$ normal goat serum (Boster Biotechnology, Wuhan, China) for $20 \mathrm{~min}$ at $37^{\circ} \mathrm{C}$. These cells were then incubated with primary antibodies (rabbit polyclonal anti-cluster of differentiation [CD]309, CD105, vWF, CD144, CD36, CD45, CD14; Boster Biotechnology) at $4{ }^{\circ} \mathrm{C}$ for $12 \mathrm{~h}$. Following three washes with PBS, the cells were further incubated with Alexa Fluor 488-labeled goat secondary antibody (Beyotime, Shanghai, China) at room temperature for $2 \mathrm{~h}$. Finally, the cells were incubated in 4, 6-diamidino-2phenylindole (DAPI) solution (Beyotime) for $5 \mathrm{~min}$. After washing with PBS, the cells were viewed under an inverted fluorescence microscope (DMI3000B, Leica, Germany). The primary antibodies were replaced with PBS in control samples. ECFCs were first incubated with DiI AcLDL (1, 1'-dioctadecyl-3, 3, 3', 3'-tetramethylindocarbocyanine [DiI]-labeled acetylated low-density lipoprotein, Thermo, MA, USA) for $4 \mathrm{~h}$ and later fixed in 1\% paraformaldehyde for $10 \mathrm{~min}$. After being washed and stained with DAPI, the cells were viewed under the inverted fluorescence microscope.

The same immunostaining procedure was followed for PBMSCs. The primary antibodies were as follows: rabbit polyclonal anti-CD105, CD106, CD90, CD34, CD14, CD45 (Boster Biotechnology), and CD73 (Proteintech, USA).

Trilineage differentiation (adipogenic, chondrogenic, and osteogenic differentiation) was conducted for PBMSCs. Cells of passage 4 were used. The induction steps and culture media were described in a previous study $^{20}$. Oil Red $\mathrm{O}$ staining was used to assess adipogenic differentiation; after fixation in $4 \%$ formalin and washing with PBS, cells were incubated in Oil Red $O$ staining solution (three parts Oil Red O stock solution: two parts de-ionized water, Leagene, Beijing, China) for $30 \mathrm{~min}$. Alizarin red staining was used to assess osteogenic differentiation; after fixation in $70 \%$ ethanol and washing with de-ionized water, cells were incubated in $0.2 \%$ Alizarin red solution (Leagene) for $10 \mathrm{~min}$. For evaluation of chondrogenic differentiation, the acquired cell mass was fixed in $4 \%$ formalin and embedded in paraffin wax for sectioning and Alcian blue staining; after being deparaffinized and re-hydrated, cells were incubated in Alcian blue solution (Cyagen, CA, USA) for $30 \mathrm{~min}$.

After 1 week in osteogenic induction medium, quantitative real-time reverse transcription polymerase chain reaction (qRT-PCR) was performed to analyze the expression of several osteogenic markers in PBMSCs. The markers included bone morphogenetic protein-2 (BMP2), alkaline phosphatase (ALP), runt-related transcription factor 2 (RUNX-2), collagen type I (COL-1), osterix (OSX), and osteocalcin (OCN). Glyceraldehyde-3phosphate dehydrogenase (GAPDH) gene expression 
Table 1 Primer sequences used for detection of osteogenic marker expression within PBMSCs

\begin{tabular}{llll}
\hline Gene & Forward primer & Reverse primer \\
\hline BMP-2 & 5'-CACCCGGCGATTCTTCTTTA-3' & 5'-AGCTTCCTGCATCTGTTCTC-3' \\
ALP & 5'-AGAAACCCTTCACTGCCATC-3' & 5'-GGTAGTTGTTGTGAGCGTAGTC-3' \\
RUNX-2 & 5'-CACTATCCAGCCACCTTTACTT-3' & 5'-TGGCAGGTAGGTATGGTAGT-3' \\
COL-1 & 5'-CGGTGGTTACGACTTTGGTTA-3' & 5'-CAGAGTGGCATCGACTTCATAG-3' \\
OSX & 5'-AGGCACGAAGAAGCCATAC-3' & 5'-CAGGTGACAGAAGCCCATT-3' & 105 \\
OCN & 5'-AGGGCCCTCACTCTTGT-3' & 5'-TGAGCCTCTGCCAGACT-3' \\
GAPDH & 5'-GGTCGGAGTGAACGGATTT-3' & 5'-TGTAGTGGAGGTCAATGAATGG-3' & 103 \\
\hline
\end{tabular}

$B M P-2$ bone morphogenetic protein-2, ALP alkaline phosphatase, RUNX-2 runt-related transcription factor $2, C O L-1$ collagen type I, OSX osterix, OCN osteocalcin, GAPDH glyceraldehyde-3-phosphate dehydrogenase

was used as the internal control. The primer sequences for these markers are listed in Table 1. The data represent the average of three independent experiments. Control cells were cultured in complete modified $\alpha$-MEM.

\section{Construction of FP scaffolds}

PLGA microspheres were prepared using a double emulsion-evaporation procedure. Briefly, $0.5 \mathrm{~g}$ PLGA (75:25, average molecular weight $\left[\mathrm{M}_{\mathrm{w}}\right]$ of $12 \mathrm{kDa}$; Daigang Biomaterials, Shandong, China) was added to a glass bottle containing $5 \mathrm{~mL}$ methylene chloride (Chinasun Specialty Products Co., Ltd, Jiangsu, China). The glass bottle was placed in a $4{ }^{\circ} \mathrm{C}$ fridge until the PLGA was fully dissolved. In the fume cabinet, $5 \mathrm{~mL}$ PLGA solution was added to a beaker followed by $200 \mu \mathrm{L}$ de-ionized water and then stirring at $1500 \mathrm{rpm}$ with a magnetic stirring apparatus for $2 \mathrm{~min}$. Next, $10 \mathrm{~mL}$ poly(vinyl alcohol) (PVA) solution ( $\mathrm{w} / \mathrm{w}=1 \%$; Sigma-Aldrich, MO, USA) was added to the oil-water mixture and then stirred at $1500 \mathrm{rpm}$ for 2-3 min. Afterward, the emulsion was poured into $400 \mathrm{~mL}$ aqueous PVA solution $(\mathrm{w} / \mathrm{w}=0.1 \%)$ and left stirring at $250 \mathrm{rpm}$ overnight. Hardened microspheres were acquired while methylene chloride evaporated. After being sieved through 50 mesh and 100 mesh, the collected microspheres were washed three times with de-ionized water and dried via vacuum drying to remove the residual methylene chloride. The morphology of microspheres was recorded by viewing them under a stereomicroscope (SMZ1000, Nikon, Tokyo, Japan) and the inverted microscope. The diameters of the prepared microspheres also were measured.

PLGA microspheres were sterilized by immersion in $75 \%$ ethanol for $2 \mathrm{~h}$ and then washed thoroughly with sterilized PBS. Next, the microspheres were soaked in complete modified $\alpha$-MEM for $48 \mathrm{~h}$ for preculture. Then PBMSCs (passage 4) were seeded onto the microspheres (in 24-well culture plates, $2 \times 10^{4}$ cells/well, $100 \mu \mathrm{L}$ PLGA microspheres/well). After being cultured in complete modified $\alpha$-MEM for 3 days, the cells were fixed in $4 \%$ paraformaldehyde for $30 \mathrm{~min}$ at $4{ }^{\circ} \mathrm{C}$. Then phalloidin (Cytoskeleton, USA) was used to stain the cytoskeleton for $20 \mathrm{~min}$. After being washed with PBS thoroughly, DAPI staining for $5 \mathrm{~min}$ was used for visualization of the nucleus. Finally, cells were observed under the inverted fluorescence microscope, as well as by scanning electron microscopy (SEM, S-3400N II, Hitachi, Tokyo, Japan) after dehydration and conductive coating.

ECFCs were infected with virus expressing red fluorescent protein (RFP; GenePharma, Shanghai, China). In brief, the culture medium for ECFCs was replaced by serum-free endothelial basal medium-2 (EBM-2, Lonza, Basel, Switzerland), and the virus suspension was added to the culture system with a multiplicity of infection (MOI) of 10. Then, polybrene (GeneChem, Shanghai, China) was added to the culture system to a final concentration of $8 \mu \mathrm{g} \mathrm{mL}^{-1}$. The EBM-2 culture medium containing virus was discarded and replaced with fresh EGM-2 after $24 \mathrm{~h}$. ECFCs of the 4th passage that had been transfected with RFP virus, as well as PBMSCs of the 4th passage were detached with trypsin/EDTA and later resuspended in fibrinogen solution (10 mg fibrinogen dissolved in $1 \mathrm{~mL}$ PBS; $50-70 \%$ protein, Sigma-Aldrich) at a ratio of $1 \times$ $10^{6}$ cells $\mathrm{mL}^{-1}$. The fibrinogen solution was coagulated into gel by adding thrombin ( $50 \mathrm{U}$ (active unit) $\mathrm{mL}^{-1}$, Sigma-Aldrich). The fibrin gel containing cells was then cultured with EGM-2mv (Lonza, Basel, Switzerland) under the condition mentioned above. After 1 week, the mixture was observed under the inverted fluorescence microscope. ECFCs cultured without PBMSCs were set as the control sample. The other conditions were the same as those used for the co-culture group.

Fibrinogen solution was pipetted to PLGA microspheres at a volume ratio of 1:2 (fibrinogen/microspheres). The fibrinogen/microspheres mixtures were then reacted with thrombin and coagulated. The FP scaffolds were observed under a stereomicroscope and inverted microscope. ImageJ $1.46 \mathrm{r}$ was used to measure the size of the pores in the scaffold. After fixation in $4 \%$ paraformaldehyde for 
$1 \mathrm{~h}$, dehydration and conductive coating, scaffolds were observed under SEM for further understanding of the structure.

\section{Induction of osteogenesis in FP scaffolds}

PBMSCs of passage 4 were suspended in fibrinogen and then added to the PLGA microspheres as mentioned above. The fibrinogen was coagulated by reaction with thrombin in vitro. The FP scaffolds were placed in the osteogenic medium (complete modified $\alpha$-MEM supplemented with $0.1 \mu \mathrm{M}$ dexamethasone, $60 \mu \mathrm{M}$ ascorbic acid, and $10 \mathrm{mM} \beta$-glycerol phosphate [Sigma-Aldrich]). After osteogenic induction for $4 \mathrm{~h}$ or 3 days, the FP scaffold samples were fixed in $4 \%$ paraformaldehyde for $24 \mathrm{~h}$. Phalloidin and DAPI staining were conducted by the method described above, and then the samples were observed under an inverted fluorescence microscope.

To analyze the effects of three-dimensional space and the rigid surface of PLGA microspheres on the osteogenic differentiation of PBMSCs, the mRNA expression of osteogenic markers BMP-2, ALP, RUNX-2, COL-1, OSX, and OCN was evaluated by qRT-PCR in PBMSCs cultured on a blank culture plate, in fibrin gel, and within the FP scaffolds. PBMSCs were seeded at a density of $8 \times 10^{4}$ cells per sample and cultured for 1 week in osteogenic medium. GAPDH gene expression was used as the internal control. The primer sequences for these markers are listed in the Table 1, and the data represent the average of three independent experiments.

Western blot analysis was performed to evaluate the protein expression of the same osteogenic markers (BMP2, ALP, COL-1, OSX, and OCN) by PBMSCs cultured in the same three culturing environments for 2 weeks in osteogenic medium. GAPDH was again used as the internal control. The following primary antibodies were used: rabbit polyclonal anti-OSX, mouse monoclonal anti-COL-I, mouse monoclonal anti-BMP-2, mouse monoclonal anti-GAPDH, mouse monoclonal anti-OCN, rabbit polyclonal anti-ALP (Abcam, Cambridge, UK). The Image 1.46r (NIH, MD, USA) and SPSS 20 (SPSS, IBM, IL, USA) software programs were used to analyze the results.

\section{Pre-vascularization of FP scaffolds}

PBMSCs were seeded into FP scaffolds with fibrin gel in advance and their osteogenic differentiation was induced as described above. After 3 weeks, the fibrin gel within the FP scaffolds was almost degraded. Then, the fibrinogen solution containing ECFCs and PBMSCs $\left(1 \times 10^{6}: 1 \times 10^{6}\right.$ cells $\mathrm{mL}^{-1}$ ) was added to FP scaffolds and coagulated. In the control group, no PBMSCs were seeded in FP scaffolds in advance, and all other conditions were the same as in the group with pre-seeding of PBMSCs. Cells were observed under the inverted microscope after 1 week.
Further characterization was conducted by histologic staining. Samples were fixed in $70 \%$ ethanol for $24 \mathrm{~h}$ and then dehydrated in a graded series of ethanol and embedded in a glycolmethacrylate resin (Technovit 7200 VLC, Heraeus Kulzer GmbH, Wehrheim, Germany). Sections $25 \mu \mathrm{m}$ in thickness were prepared using the EXACT system (EXACT, Germany) and stained with Mayer's hematoxylin solution (Leagene) followed by ponceau-acid fuchsin solution (Leagene).

\section{In vivo evaluation of bone regeneration}

Adult healthy New Zealand white rabbits, weighing $4.0-4.5 \mathrm{~kg}$, were used to create a mandibular bone defect model. The rabbits were randomly assigned to three groups: (1) blank control (BC): bone defect was created without implantation of a scaffold; (2) FP scaffold group (FP): bone defect was created and cell-free FP scaffold was implanted; and (3) cell-laden FP scaffold group (CFP group): bone defect was created and FP scaffolds preseeded with PBMSCs and ECFCs were implanted. The process was based on a published protocol ${ }^{9}$. Briefly, after anesthesia, a midline incision was made on the submental area. Then, the tissue was dissected in layers until the buccal side of the mandible was exposed. An 8-mm cylindrical full-thickness bone defect was created in the mandible with a trephine bur. Then, the corresponding implants were placed in the defects and their shapes were prepared to adapt the defects. After the operation, the muscle, fascia, and skin were closed in layers. The rabbits were fed a normal diet, and at 6 and 12 weeks after operation, the rabbits were euthanized for collection of mandible samples.

\section{Micro-computed tomography (CT) examination}

The harvested mandible samples were analyzed by micro-CT (vivaCT80, Zurich, Switzerland), with scanning parameters of $55 \mathrm{kVp}$ and $145 \mu \mathrm{A}$. The original bone defect was included in each scan. Data were analyzed using CT Analyser (1.15.4.0, Bruker microCT, Kontich, Belgium), DataViewer (1.5.1.2, Bruker microCT), and Ctvox (3.0.0.0, Bruker microCT). The bone volume/total volume $(\mathrm{BV} / \mathrm{TV})$ for the bone defect region was calculated.

\section{Statistical analysis}

Statistical differences between two groups were analyzed by Student's $t$-test. One-way analysis of variance (ANOVA) followed by least-significant difference test was adopted to analyze differences between more than two groups. All quantitative data reported represent the mean of at least three independent samples. A $p$-value $<0.05$ was considered significant. SPSS 20 was used for statistical analyses. 


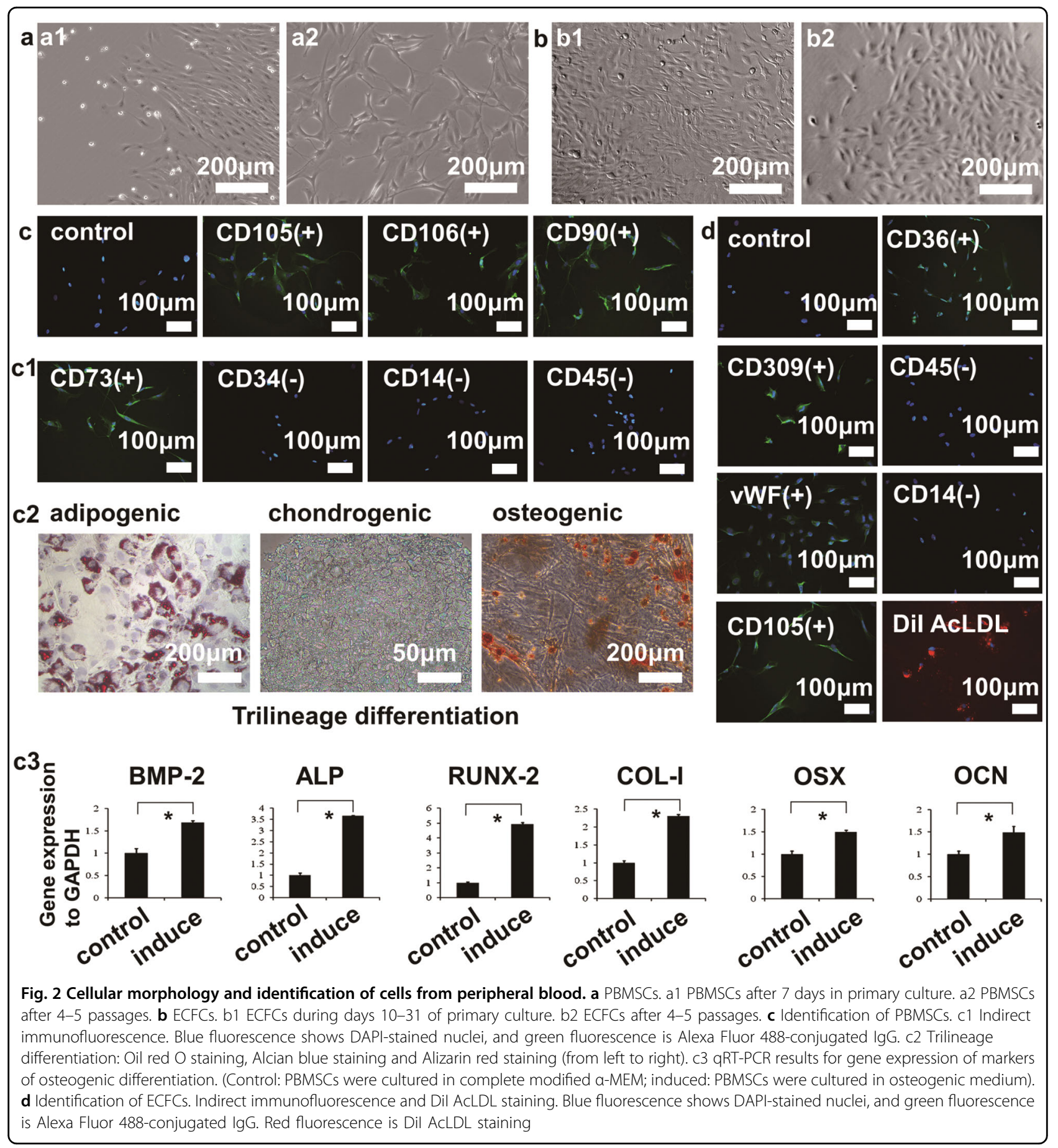

\section{Results and discussion}

Isolation of PBMSCs and ECFCs from peripheral blood

Cells were collected from peripheral blood and isolated. After 7 days in culture, some adherent cells with a spindle shape (Fig. 2, a1) had formed clusters. During days 7-21 in culture, these cells proliferated rapidly and maintained a stable morphology after 4-5 passages (Fig. 2, a2). Another cell type was observed in the primary culture after
10-31 days. The morphology of these cells matched the "cobblestone-like" shape (Fig. 2, b1), which made them easy to be identify ${ }^{19}$. Typically, they appeared among the clusters of the other cell type mentioned above and had a stable morphology after 4-5 passages (Fig. 2, b2).

Phenotypic identification of the spindle-shaped cells revealed that the cells were: CD105 (+), CD106 (+), CD73 $(+), \operatorname{CD} 90(+), \operatorname{CD} 34(-), \operatorname{CD} 14(-)$, and CD45 (-) 

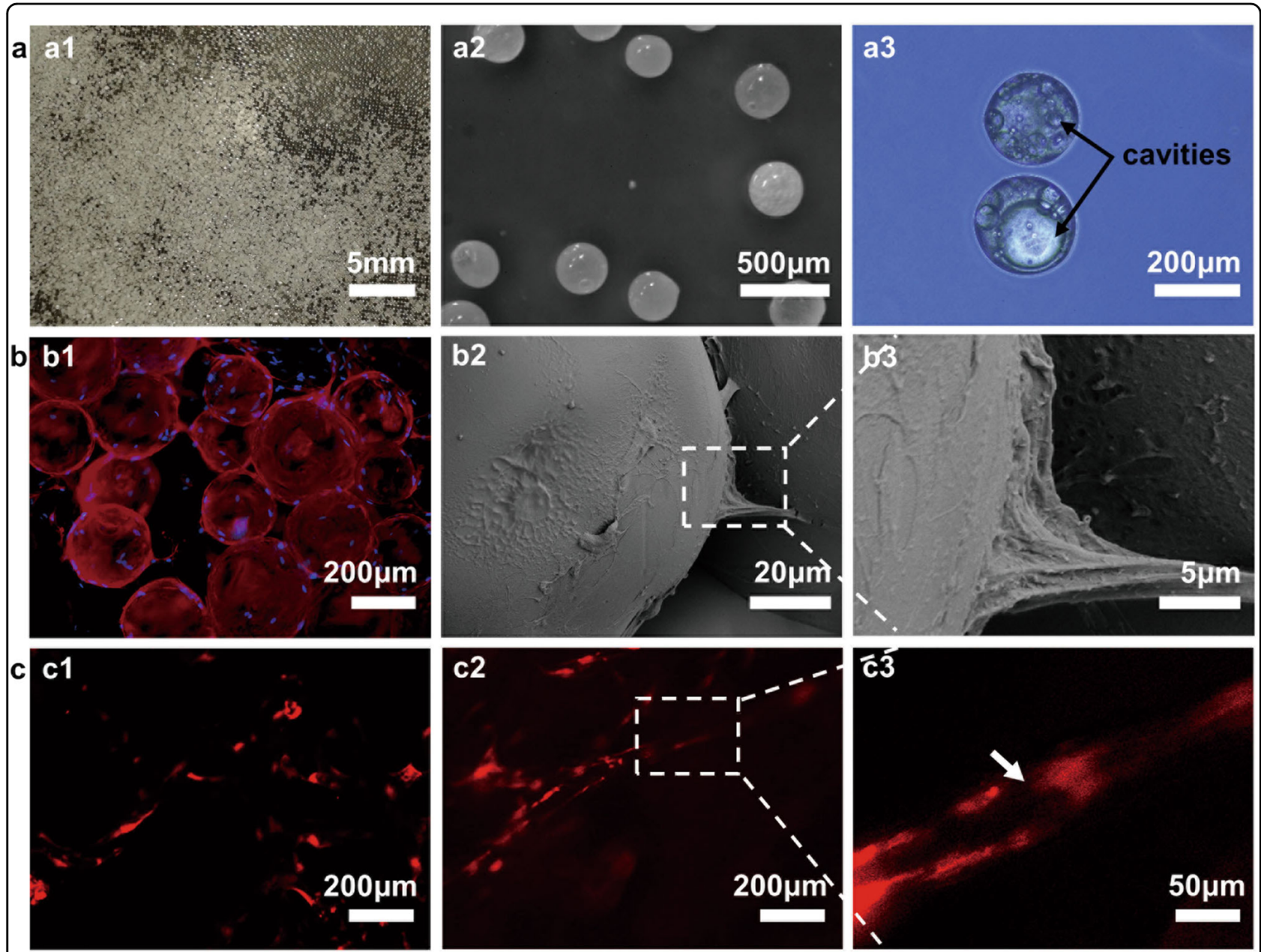

Fig. 3 PLGA microspheres and fibrin gel. a Morphology and structure of PLGA microspheres. a1 Microspheres after vacuum freeze drying. a2 Stereomicroscopy image of translucent PLGA microspheres. a3 Hollow microspheres observed under an inverted microscope. $\mathbf{b}$ Cells adhered on the surface of PLGA microspheres. b1 Fluorescence staining. Blue fluorescence is DAPI, red fluorescence is phalloidin. b2-3 SEM images show cells adhered on microspheres. c Capillary-like structures formed in the fibrin gel. c1 Control group: ECFCs cultured without PBMSCs. C2 Co-culture group: ECFCs cultured with PBMSCs. C3 Amplified image of the co-culture group: capillary-like structures with lumens were observed (white arrow). Red fluorescence indicates expression of red fluorescent protein (RFP)

(Fig. 2, c1). These results are consistent with the identification criteria for $\mathrm{MSCs}^{34}$, and the MSCs from blood ${ }^{20}$. Staining with Oil red $\mathrm{O}$, Alcian blue and Alizarin red for trilineage (adipogenic, chondrogenic, and osteogenic, respectively) differentiation were positive, which further confirmed that the cells were MSCs (Fig. 2, c2). qRT-PCR analysis showed significant upregulation of gene markers of osteogenic differentiation (BMP-2, ALP, RUNX-2, COL-1, OSX, and OCN) after the cells were exposed to osteogenic medium for 7 days (Fig. 2, c3). Together, the results above indicate that the spindle-shaped cells were PBMSCs.

Phenotypic identification of the cobblestone-like cells revealed that the cells were CD309 $(+)$, vWF $(+)$, CD105 (+), CD36 (+), CD45 (-), and CD14 (-), which has been shown to be representative of ECFCs by previous studies ${ }^{33,35,36}$. The cells also had the capacity to incorporate DiI AcLDL, which further confirmed that these cells were ECFCs (Fig. 2d) ${ }^{33}$.

\section{Construction of FP scaffold}

PLGA microspheres were successfully prepared by a double emulsion-evaporation procedure and dried by vacuum freezing (Fig. 3, a1). The microspheres were translucent under a stereomicroscope (Fig. 3, a2). The shape of spheres had the minimum anisotropy, which ensured that cells were cultured in a relatively uniform environment within the prepared scaffolds. The hollow nature of the spheres afforded the potential for drug loading and controlled drug release (Fig. 3, a3).

Attachment of PBMSCs to PLGA microspheres was observed under fluorescence microscopy (Fig. 3, b1) and SEM (Fig. 3, b2-3). In fact, the microspheres were 


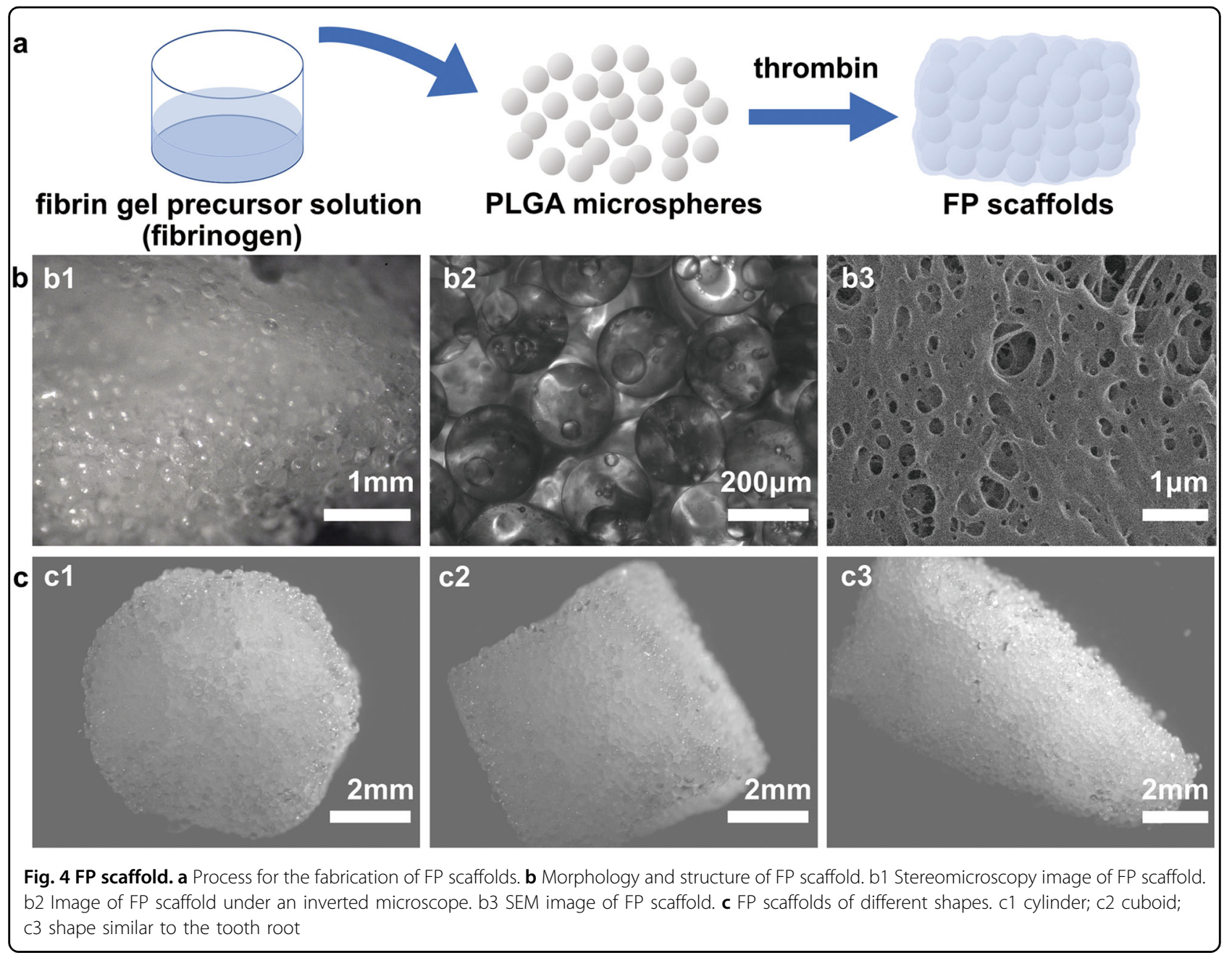

surrounded by cells, and cells could even adhere to two adjacent microspheres. These findings show that the microspheres could serve as microcarriers by providing a surface for cell adhesion ${ }^{26}$.

Evaluation of the size of the PLGA microspheres showed that the diameter of the microspheres was $243.34 \pm 31.79 \mu \mathrm{m}$, and the inner pore size of stacked microspheres ranged from 100 to $200 \mu \mathrm{m}$, which is adequate for cell growth and migration. Moreover, some studies showed scaffolds with a pore size of $100-250 \mu \mathrm{m}$ or larger are suitable for bone regeneration ${ }^{37}$.

Elongated capillary-like structures were found in the fibrin gel after co-culture of ECFCs and PBMSCs for 7 days. In addition, lumens were found in these structures (Fig. 3, c2-3). By comparison, the control group in which ECFCs were cultured without PBMSCs showed no evidence of capillary-like structures (Fig. 3, c1). These results showed the capability of fibrin gel and PBMSCs, which helps to achieve pre-vascularization of scaffolds. Previous studies showed that endothelial cells (ECFCs in our study) may form capillary-like structures spontaneously in gel materials (fibrin gel in our study), and MSCs (PBMSCs in our study) were used as pericytes ${ }^{14,18}$. In summary, with the co-culture of ECFCs and PBMSCs, capillary-like structures formed in the fibrin gel.

Fibrin gel precursor solution (fibrinogen) was added to the PLGA microspheres and coagulated to form the FP scaffold (Fig. 4a). Under a stereomicroscope, the PLGA microspheres were cemented together by the fibrin gel (Fig. 4, b1). These microspheres were stacked together with interconnected pores among them under an inverted microscope (Fig. 4, b2), and the pores were filled with fibrin gel. SEM showed that the fibrin gel in the pores possessed a porous net-like structure (Fig. 4, b3).

Addition of the fibrin gel also made the FP scaffold material easy to shape, which is important for filling unique bone defects. FP scaffolds can be easily prepared in a customized shape to match a defect or the shape of a tooth root (Fig. 4c). 


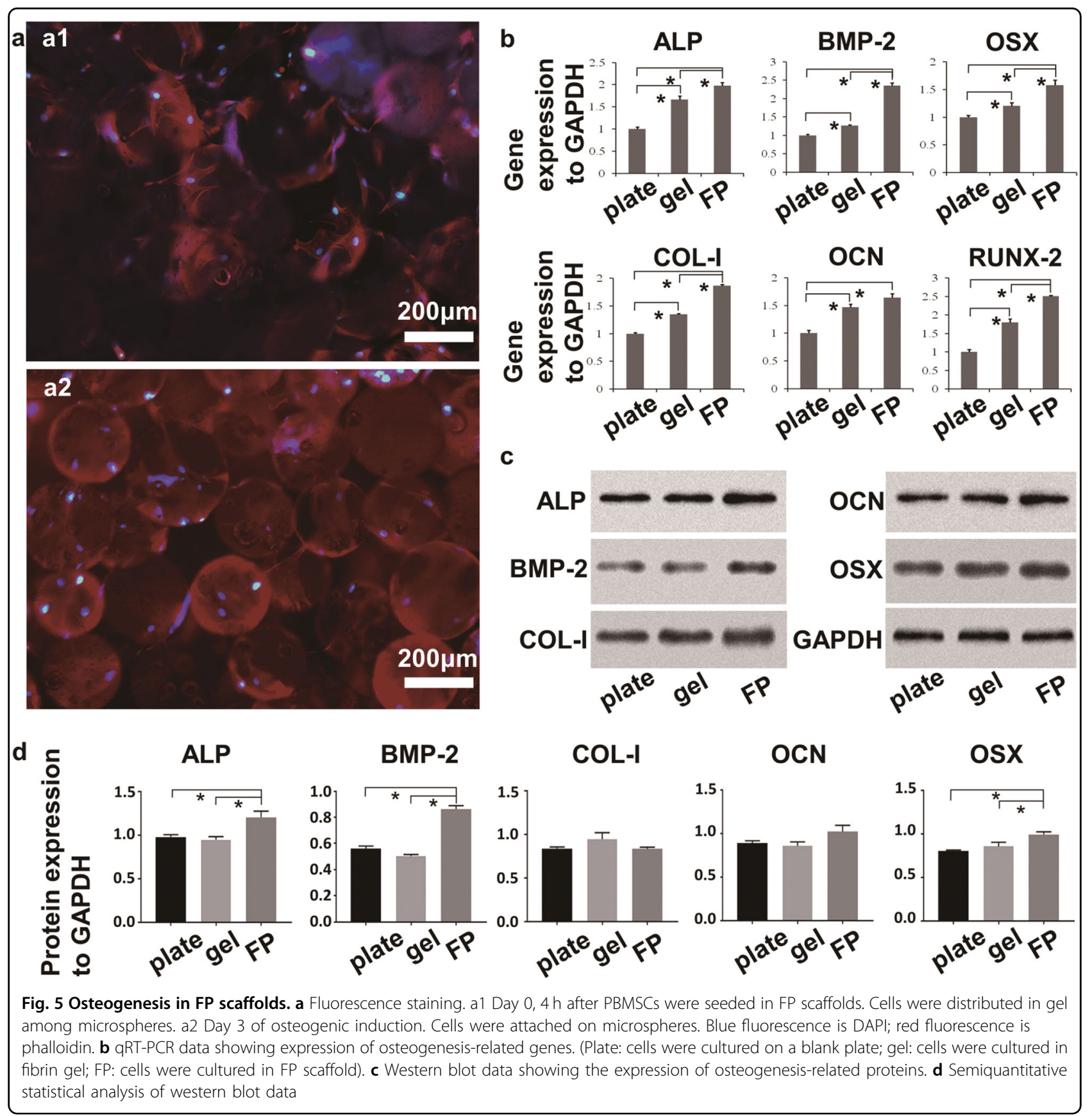

\section{Osteogenic differentiation of PBMSCs in FP scaffolds}

During the period of osteogenic induction, fluorescence staining was used to locate the cells that had been seeded within fibrin gel in the FP scaffolds. Cells exhibiting the red fluorescence of phalloidin had adhered to the surface of PLGA microspheres by the third day of osteogenic induction, while almost no cells were left in the space among microspheres (Fig. 5a). This may be the consequence of migration caused by the preference of PBMSCs to adhere to the rigid substrate in FP scaffolds ${ }^{38}$. PBMSCs were seeded within the fibrin gel precursor solution, which would coagulate rapidly upon addition of thrombin. The mass fraction of water in fibrin gel is $99 \%$, and the cells in the matrix of gel can migrate freely rather than sinking, which commonly occurs due to gravity when cells are seeded with a liquid culture medium, leading to heterogeneous dispersal in three-dimensional scaffolds ${ }^{39}$.

The expression levels of osteogenic differentiationrelated genes (BMP-2, ALP, RUNX-2, COL-1, OSX, and OCN) differed significantly among PBMSCs cultured in the three different environments (tissue culture plate, 


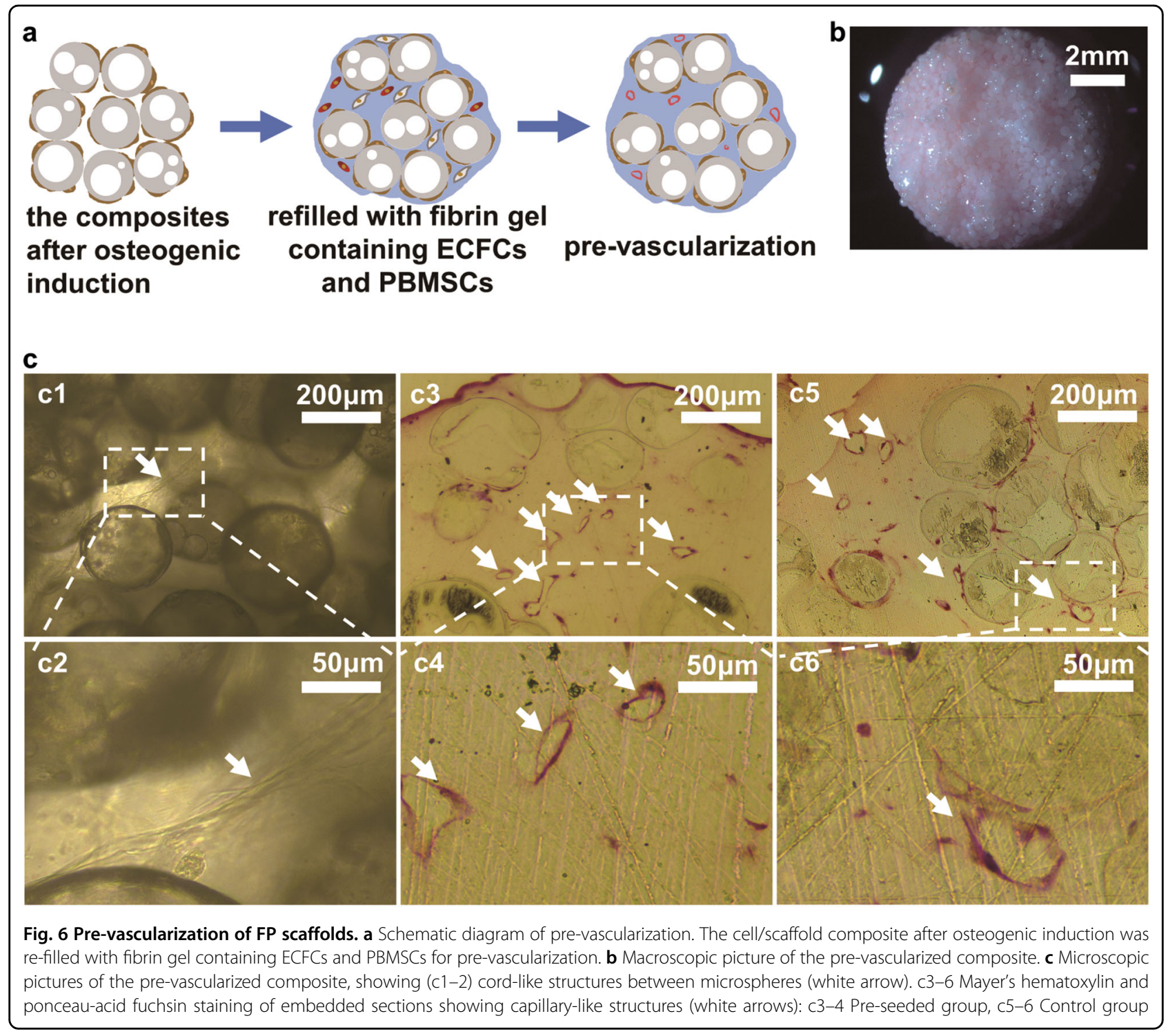

fibrin gel, and FP scaffold; except the expression of OCN between fibrin gel and FP scaffold; Fig. 5b). Higher expression of these genes was detected in gel than the plate group, and the highest expression was detected in the FP group. The relative expression levels of proteins (BMP-2, ALP, COL-1, OSX, and OCN) showed a similar trend (Fig. 5c). Semiquantitative statistical analyses indicated that the expression levels of the protein markers (except OCN and COL-1, which did not significantly differ among three groups) in FP were significantly higher than those observed in gel and plate (Fig. 5d). Based on these results above, this upregulated expression of PBMSCs in gel and FP may be attributed to the ideal microenvironment for osteogenic differentiation provided by the three-dimensional space ${ }^{22}$. FP showed the highest protein expression levels. This might be due to the rigid surface of the PLGA microspheres. Wang et al. ${ }^{26}$ reported that a rigid substrate can provide a better cell-adhesive surface for anchorage-dependent cells such as MSCs and promote osteogenic differentiation.

\section{Pre-vascularization of FP scaffolds}

After 3 weeks in culture in osteogenic medium, the fibrin gel within the FP scaffolds was almost degraded, and the cell/scaffold composite was then refilled with fibrin gel containing ECFCs and PBMSCs for prevascularization (Fig. 6a). In the prepared composite (Fig. 6b), cord-like structures were found among the PLGA microspheres (Fig. 6, c1-2) by 1 week after the scaffolds had been filled with the fibrin gel containing ECFCs and PBMSCs. Upon staining of embedded sections of the scaffolds, plenty of capillary-like lumens with generally irregular or oval shapes were found throughout FP scaffolds. Most were distributed in the space among the 

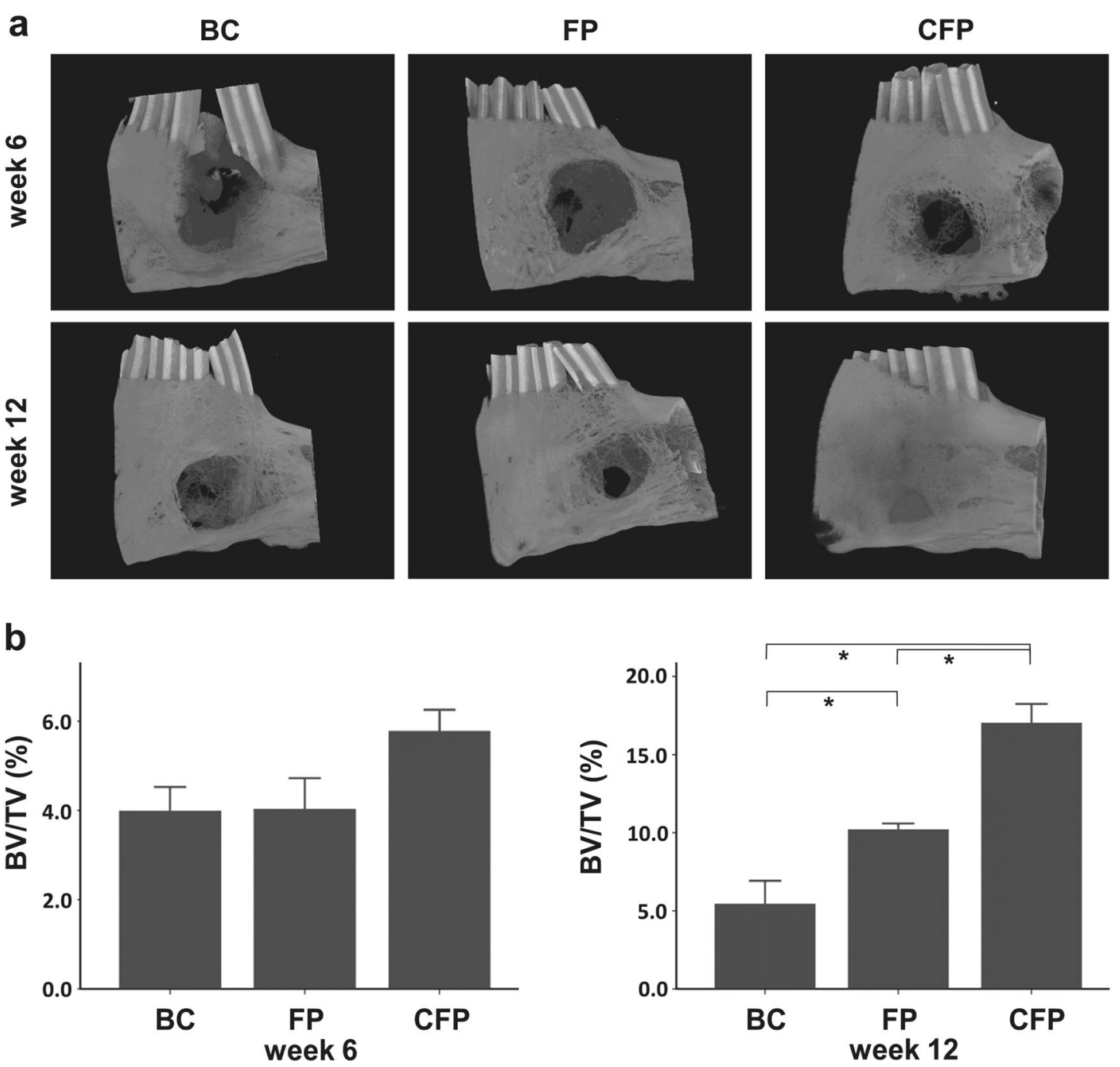

Fig. 7 Micro-CT evaluation of in vivo bone growth within harvested mandible samples. a Three-dimensional reconstructed images at week 6 and 12. (BC: Blank control, bone defect without implant; FP: Bone defect implanted with FP scaffold; CFP: Bone defect implanted with cell-laden FP scaffolds). $\mathbf{b}$ Results of quantitative analyses of samples harvested at weeks 6 and 12. The symbol * denotes a statistical significance between two groups $(p<0.05)$

microspheres (Fig. 6, c3-4). The diameter of the capillarylike structures was about $30 \mu \mathrm{m}$, which is close to the size of natural capillaries in vivo ${ }^{40}$. As indicated by previous studies, MSCs and osteoblasts can secrete angiogenic cytokines such as vascular endothelial growth factor via paracrine signaling, which may influence vascularization, promoting the formation, and maturation of vessels ${ }^{14,41}$. The PBMSCs in our study may play roles similar to those of MSCs and osteoblasts, respectively. Moreover, in the control samples, in which no PBMSCs were pre-seeded, capillary-like structures were also observed (Fig. 6, c5-6). So, the osteogenic differentiation of pre-seeded PBMSCs induced by osteogenic medium did not seem to interfere with the pre-vascularization established by the co-culture of ECFCs and PBMSCs.
ECFCs play an important role in angiogenesis ${ }^{42}$, and the capillary-like structures they form can anastomose with the host vasculature quickly in vivo ${ }^{15}$. The co-culture strategy closely mimicked the in vivo angiogenesis process $^{43}$. After implantation, the capillary-like structures formed by ECFCs would not only be anastomosed with the host vasculature, but also stimulate the rapid growth of natural capillaries into the scaffold from the surrounding tissue ${ }^{16}$.

Previous studies have also shown that PBMSCs can participate in both blood vessel formation and bone repair $^{41,44}$. To reduce interference between these two roles and avoid additional complex interactions, the induction of osteogenesis and the pre-vascularization of the scaffolds were conducted separately by 
culturing cell-laden scaffolds under different conditions sequentially.

\section{In vivo bone defect repair using pre-vascularized FP scaffolds}

According to the three-dimensional reconstruction results from micro-CT (Fig. 7a), new bone formation was observed in all the three groups (BC group, FP group, and CFP group) at the region of the bone defect. However, the $\mathrm{BC}$ group presented the least amount of bone growth at both week 6 and week 12. Bone formation in the FP group was better, but the most bone growth was observed in the CFP group. The results of further quantitative analysis involving calculation of the BV/TV (Fig. 7b) was consistent with the results of micro-CT examination. The CFP group had the highest BV/TV value, and statistically significant differences in the BV/TV were observed among three groups at week 12 (but not at week 6). In summary, the CFP group led to superior bone regeneration compared with that observed in the FP group and the $\mathrm{BC}$ group, indicating that this type of pre-vascularized scaffold might promote bone healing at defect sites.

\section{Conclusion}

Cells of different types prefer matrices of different rigidity. The assembly of components of differing rigidity in a functional composite in a manner that allows different cell types to maintain their phenotype represents a significant challenge. In the present study, two materials with different rigidity, PLGA and fibrin gel, were integrated into an ordered and functional scaffold. PLGA microspheres provided a sufficient and rigid inner surface for PBMSC attachment, whereas fibrin gel filled the interconnected pores among the microspheres and supported the formation of capillary-like structures from ECFCs co-cultured with PBMSCs. To achieve the purposes of both vascularization and bone regeneration, prevascularization and induction of osteogenic differentiation can be implemented separately under different conditions in FP scaffolds. The goal of pre-vascularization is to facilitate rapid anastomosis with the host vasculature for the transport of oxygen and nutrients to all cells within a scaffold, which may lead to better bone regeneration in the future.

\section{Acknowledgements}

J.L. and G.C. contributed equally to this work. The authors gratefully acknowledge support from The National Key Research and Development Project of China (2016YFA0201704/2016YFA0201700) and the Priority Academic Program Development of Jiangsu Higher Education Institutions (PAPD, 2014-37).

\section{Author details}

${ }^{1}$ Jiangsu Key Laboratory of Oral Diseases, Nanjing Medical University, 210029 Nanjing, China. ${ }^{2}$ Key Laboratory of Clinical and Medical Engineering, School of Biomedical Engineering, Department of Basic Medical Sciences, Nanjing Medical University, 210029 Nanjing, China. ${ }^{3}$ State Key Laboratory of
Bioelectronics, Jiangsu Key Laboratory of Biomaterials and Devices, School of Biological Science and Medical Engineering, Southeast University, 210009 Nanjing, China

Conflict of interest

The authors declare that they have no conflict of interest.

\section{Publisher's note}

Springer Nature remains neutral with regard to jurisdictional claims in published maps and institutional affiliations.

Received: 8 October 2017 Revised: 13 June 2018 Accepted: 2 July 2018. Published online: 28 August 2018

\section{References}

1. Roberts, S. J. et al. The combined bone forming capacity of human periosteal derived cells and calcium phosphates. Biomaterials 32, 4393-4405 (2011).

2. Lohmann, P. et al. Bone regeneration induced by a 3D architectured hydrogel in a rat critical-size calvarial defect. Biomaterials 113, 158-169 (2017).

3. Torres-Rendon et al. Bioactive gyroid scaffolds formed by sacrificial templating of nanocellulose and nanochitin hydrogels as instructive platforms for biomimetic tissue engineering. Adv. Mater. 27, 2989-2995 (2015).

4. Rutz, A. L., Hyland, K. E., Jakus, A. E., Burghardt, W. R. \& Shah, R. N. A multimaterial bioink method for 3D printing tunable, cell-compatible hydrogels. Adv. Mater. 27, 1607-1614 (2015).

5. Hashmi, B. et al. Developmentally-inspired shrink-wrap polymers for mechanical induction of tissue differentiation. Adv. Mater. 26, 3253-3257 (2014).

6. Grayson, W. L. et al. Stromal cells and stem cells in clinical bone regeneration. Nat. Rev. Endocrinol. 11, 140-150 (2015).

7. Smith, B. D. \& Grande, D. A. The current state of scaffolds for musculoskeletal regenerative applications. Nat. Rev. Rheumatol. 11, 213-222 (2015).

8. Lovett, M., Lee, K., Edwards, A. \& Kaplan, D. L. Vascularization strategies for tissue engineering. Tissue Eng. Part B Rev. 15, 353-370 (2009).

9. Shah, S. R. et al. A composite critical-size rabbit mandibular defect for evaluation of craniofacial tissue regeneration. Nat. Protoc. 11, 1989-2009 (2016).

10. Kearney, J. B., Kappas, N. C., Ellerstrom, C., DiPaola, F. W. \& Bautch, V. L. The VEGF receptor flt-1 (VEGFR-1) is a positive modulator of vascular sprout formation and branching morphogenesis. Blood 103, 4527-4535 (2004).

11. Bersini, S. et al. Human in vitro 3D co-culture model to engineer vascularized bone-mimicking tissues combining computational tools and statistical experimental approach. Biomaterials 76, 157-172 (2016).

12. Wray, L. S., Tsioris, K., Gi, E. S., Omenetto, F. G. \& Kaplan, D. L. Slowly degradable porous silk microfabricated scaffolds for vascularized tissue formation. Adv. Funct. Mater. 23, 3404-3412 (2013).

13. Sekine, $\mathrm{H}$. et al. In vitro fabrication of functional three-dimensional tissues with perfusable blood vessels. Nat. Commun. 4, 1399 (2013).

14. Novosel, E. C., Kleinhans, C. \& Kluger, P. J. Vascularization is the key challenge in tissue engineering. Adv. Drug Deliv. Rev. 63, 300-311 (2011).

15. Kang, K. T., Allen, P. \& Bischoff, J. Bioengineered human vascular networks transplanted into secondary mice reconnect with the host vasculature and reestablish perfusion. Blood 118, 6718-6721 (2011).

16. Unger, R. E. et al. The rapid anastomosis between prevascularized networks on silk fibroin scaffolds generated in vitro with cocultures of human microvascular endothelial and osteoblast cells and the host vasculature. Biomaterials 31, 6959-6967 (2010)

17. Rao, R. R. \& Stegemann, J. P. Cell-based approaches to the engineering of vascularized bone tissue. Cytotherapy 15, 1309-1322 (2013).

18. Chen, Y. C. et al. Functional human vascular network generated in photocrosslinkable gelatin methacrylate hydrogels. Adv. Funct. Mater. 22, 2027-2039 (2012).

19. Ingram, D. A., Caplice, N. M. \& Yoder, M. C. Unresolved questions, changing definitions, and novel paradigms for defining endothelial progenitor cells. Blood 106, 1525-1531 (2005).

20. Tondreau, T. et al. Mesenchymal stem cells derived from CD133-positive cells in mobilized peripheral blood and cord blood: proliferation, Oct4 expression, and plasticity. Stem Cells 23, 1105-1112 (2005). 
21. Shi, Z. et al. Electroconductive natural polymer-based hydrogels. Biomaterials 111, 40-54 (2016)

22. Zhang, Z. et al. Nasal ectomesenchymal stem cells: multi-lineage differentiation and transformation effects on fibrin gels. Biomaterials 49, 57-67 (2015).

23. Singh, R. K., Seliktar, D. \& Putnam, A. J. Capillary morphogenesis in PEGcollagen hydrogels. Biomaterials 34, 9331-9340 (2013).

24. Riemenschneider, S. B. et al. Inosculation and perfusion of pre-vascularized tissue patches containing aligned human microvessels after myocardial infarction. Biomaterials 97, 51-61 (2016).

25. Li, Z. et al. Differential regulation of stiffness, topography, and dimension of substrates in rat mesenchymal stem cells. Biomaterials 34, 7616-7625 (2013).

26. Wang, C. et al. The control of anchorage-dependent cell behavior within a hydrogel/microcarrier system in an osteogenic model. Biomaterials $\mathbf{3 0}$ 2259-2269 (2009).

27. Kang, H. W. et al. A 3D bioprinting system to produce human-scale tissue constructs with structural integrity. Nat. Biotechnol. 34, 312-319 (2016).

28. Wang, M. O. et al. Evaluating 3D-printed biomaterials as scaffolds for vascularized bone tissue engineering. Adv. Mater. 27, 138-144 (2015).

29. Gaharwar, A. K. et al. Shear-thinning nanocomposite hydrogels for the treatment of hemorrhage. ACS Nano. 8, 9833-9842 (2014).

30. Li, Y., Meng, H., Liu, Y. \& Lee, B. P. Fibrin gel as an injectable biodegradable scaffold and cell carrier for tissue engineering. ScientificWorldJournal 2015 685690 (2015).

31. Wang, $H$. et al. Hyaluronic acid-decorated dual responsive nanoparticles of Pluronic F127, PLGA, and chitosan for targeted co-delivery of doxorubicin and irinotecan to eliminate cancer stem-like cells. Biomaterials 72, 74-89 (2015).

32. Landau, S. et al. Tropoelastin coated PLLA-PLGA scaffolds promote vascular network formation. Biomaterials 122, 72-82 (2017).

33. Lin, Y., Weisdorf, D. J., Solovey, A. \& Hebbel, R. P. Origins of circulating endothelial cells and endothelial outgrowth from blood. J. Clin. Invest. 105, 71-77 (2000).
34. Dominici, M. et al. Minimal criteria for defining multipotent mesenchymal stromal cells. The International Society for Cellular Therapy position statement Cytotherapy 8, 315-317 (2006).

35. Martin-Ramirez, J., Hofman, M., van den Biggelaar, M., Hebbel, R. P. \& Voorberg J. Establishment of outgrowth endothelial cells from peripheral blood. Nat. Protoc. 7, 1709-1715 (2012).

36. Jerkic, M. \& Letarte, M. Increased endothelial cell permeability in endoglindeficient cells. FASEB J. 29, 3678-3688 (2015).

37. Jiang, T., Abdel-Fattah, W. I. \& Laurencin, C. T. In vitro evaluation of chitosan/ poly(lactic acid-glycolic acid) sintered microsphere scaffolds for bone tissue engineering. Biomaterials 27, 4894-4903 (2006).

38. Ueki, A. \& Kidoaki, S. Manipulation of cell mechanotaxis by designing curvature of the elasticity boundary on hydrogel matrix. Biomaterials 41, 45-52 (2015).

39. Swartz, D. D., Russell, J. A. \& Andreadis, S. T. Engineering of fibrin-based functional and implantable small-diameter blood vessels. Am. J. Physiol. Heart Circ. Physiol. 288, H1451-H1460 (2005).

40. Heller, M. et al. Tissue engineered pre-vascularized buccal mucosa equivalents utilizing a primary triculture of epithelial cells, endothelial cells and fibroblasts. Biomaterials 77, 207-215 (2016).

41. Rustad, K. C. et al. Enhancement of mesenchymal stem cell angiogenic capacity and stemness by a biomimetic hydrogel scaffold. Biomaterials $\mathbf{3 3}$, 80-90 (2012)

42. Margheri, F. et al. Endothelial progenitor cell-dependent angiogenesis requires localization of the full-length form of UPAR in caveolae. Blood 118, 3743-3755 (2011).

43. Kirkpatrick, C. J., Fuchs, S. \& Unger, R. E. Co-culture systems for vascularization-learning from nature. Adv. Drug Deliv. Rev. 63, 291-299 (2011).

44. Jones, E. \& McGonagle, D. Human bone marrow mesenchymal stem cells in vivo. Rheumatol. (Oxf.). 47, 126-131 (2008). 